ORIGINAL

\title{
Evaluación de la reproducción inducida de nicuro Pimelodus blochii (Telostei: Pimelodidae) utilizando diferentes inductores hormonales
}

\author{
Evaluation of induced breeding of Pimelodus blochii nicuro \\ (telostei, pimelodidae) using different inducing hormone
}

\author{
Juan Ramírez M, ${ }^{1 *}$ M.Sc, Cristian Cifuentes $C,{ }^{1}$ MVZ, Yinet Parrado $S,{ }^{1}$ MVZ, \\ Mónica Avilés B, ${ }^{1}$ Biólogo.
}

\begin{abstract}
${ }^{1}$ Corporación Centro de Desarrollo Tecnológico Piscícola Surcolombiano - ACUAPEZ. Grupo de Investigación en Acuicultura Estratégica. Neiva, Huila, Colombia. *Correspondencia: juanantonioramirez.merlano@gmail.com.
\end{abstract}

Recibido: Abril de 2011; Aceptado: Febrero de 2012.

\section{RESUMEN}

Objetivo. Evaluar el efecto de dos inductores hormonales en la reproducción inducida de nicuro Pimelodus blochii. Materiales y métodos. Para los procesos experimentales fueron utilizados adultos sexualmente maduros, sometidos a tres tratamientos aplicados vía intramuscular, en dosis única de $0.25 \mathrm{~mL} / \mathrm{kg}$ Ovaprim \& (OVAP) (T1), $0.5 \mathrm{~mL} / \mathrm{kg}$ de OVAP (T2) y $6.25 \mathrm{mg} / \mathrm{kg}$ de Extracto de Hipófisis de Carpa (EHC) (T3), para este último tratamiento la inyección fue dividida en 20 y $80 \%$, con un intervalo de $12 \mathrm{~h}$ entre aplicaciones. Previo a la extracción de los gametos, los animales fueron tranquilizados por inmersión en una solución de Metanosulfonato de Tricaina ( $90 \mathrm{mg} / \mathrm{L}$ ). El desempeño reproductivo fue evaluado mediante el índice de ovulación (hembras ovuladas/hembras tratadas), fecundidad absoluta (Fa) (ovocitos/hembra), fecundidad relativa (Fr) en función del número de ovocitos desovados por gramo de peso. La fecundación se realizó en seco y seis horas post-fecundación (HPF) se determinó

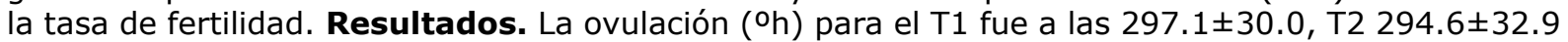
y T3 $247.3 \pm 13.1$ h. En todos los tratamientos se obtuvieron hembras ovuladas, donde los mayores índices de ovulación fueron obtenidos con Ovaprim \& (T1 y T2) con 36.4 y 50\%, respectivamente. Las tasas de fecundación obtenidas fueron mayores a un $50 \%$, para el tratamiento 1 y 2 , con valores de 74.5 y $32.7 \%$, respectivamente. Conclusiones. El uso de inductores hormonales puede ser efectivo para garantizar la reproducción inducida del nicuro, en dosis única de 0.25 y $0.5 \mathrm{~mL} / \mathrm{kg}$ de Ovaprim .

Palabras clave: Gametos, Pimelodus blochii, reproducción sexual (Fuente:CAB). 


\begin{abstract}
Objective. Evaluate the effect of two hormonal inducers in induced breeding of nicuro Pimelodus blochii. Materials and methods. The experimental process used sexually mature adults exposed to three intramuscular treatments, a single dose of $0.25 \mathrm{~mL} / \mathrm{g}$ Ovaprim $\AA$ (OVAP) (T1), $0.5 \mathrm{~mL} / \mathrm{kg}$ of OVAP (T2) and $6.25 \mathrm{mg} / \mathrm{kg}$ of pituitary carp extract (EPC) (T3), for the latter treatment the injection was divided into 20 and $80 \%$, with an interval of 12 hours between applications. Prior to the removal of gametes, the animals were tranquilized by immersion in a Tricaine Methanesulfonate $(90 \mathrm{mg} / \mathrm{L})$ solution. Reproductive performance was evaluated using ovulation rates (females ovulated/treated females), fertility ( $\mathrm{Fa}$ ) (eggs/female), relative fertility ( $\mathrm{Fr}$ ) depending on the number of eggs spawned per gram of weight. The dry method of fertilization was used and the fertility rate was determined six

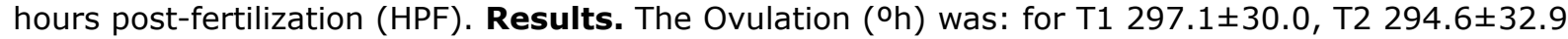
and T3 $247.3 \pm 13.1 \mathrm{~h}$. Ovulated females were obtained in all treatments, where the highest rates of ovulation were obtained with Ovaprim $\AA$ ( $T 1$ and $\mathrm{T} 2)$ with 36.4 and $50 \%$ respectively. Fertilization rates were higher than $50 \%$ for treatments 1 and 2, with values of 74.5 and $32.7 \%$ respectively. Conclusions. The use of hormonal inducers may be effective to ensure nicuro induced reproduction with a single dose of 0.25 and $0.5 \mathrm{~mL} / \mathrm{kg}$ Ovaprim $^{\circledR}$.
\end{abstract}

Key words: Gametes, Pimelodus blochii, sexual reproduction (Source:CAB).

\section{INTRODUCCIÓN}

La especie Pimelodus blochii Valenciennes, 1840, conocida comúnmente como: nicuro, chorrosco, barbudo (Pimelodidae), se clasifica dentro de los pequeños bagres y se distribuye en los ríos Magdalena, Cauca, Sinú, San Jorge, Cesar, Atrato y Baudó (1). Esta especie es importante para la pesca artesanal en la cuenca del río Magdalena (2) y es identificada como una especie migratoria (3), cuyo comportamiento trófico y reproductivo, al igual que el de otras especies de su género, está asociado en parte, con la dinámica hidrológica de los sistemas donde se presenta (4). Esto permite considerar a esta especie como un ejemplar óptimo para explotaciones piscícolas regionales y modelo ideal para ensayos de reproducción en cautiverio (5). De acuerdo con López-Casas y Jiménez-Segura (6) el nicuro para el caso de las hembras maduras presentan tallas entre los 13.2 y $21.6 \mathrm{~cm}$ de longitud estándar, siendo el mes de octubre el de mayor prevalencia de hembras maduras. Sin embargo, la mayoría de las especies de peces tropicales de agua dulce, de interés comercial como el nicuro $P$. blochii, son reofilicos, y por lo tanto precisan migrar para poder reproducirse, evento asociado a los periodos de lluvia, provocando disfunciones reproductivas cuando son mantenidos en cautiverio (7). Estas disfunciones reproductivas, son el resultado probablemente de la combinación del estrés generado por el cautiverio y la pérdida de las condiciones ambientales apropiadas para su reproducción natural (8).

Informaciones sobre el comportamiento reproductivo de nicuro, en su hábitat natural y en ambientes confinados son escasas y para que su producción de progenies tenga éxito es necesario el uso de sustancias hormonales para inducir la vitelogénesis, maduración, ovulación y finalmente el desove. Sustancias inductoras como Extracto de Hipófisis de Carpa (EHC) y análogos de la hormona liberadora de

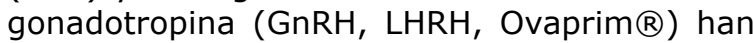
dado resultados positivos en la inducción a la maduración final y ovulación de las especies nativas (9). Sin embargo, el EHC presenta algunas desventajas, como su variabilidad en la cantidad y calidad de hormonas presentes y su alto costo en el comercio. En contraste la hormona liberadora de gonadotropina con un bloqueador de los receptores de D2 de dopamina, Ovaprim ${ }^{\circledR}$ presenta ventajas, como la no generación de respuesta inmunológica, induce la liberación de GtH endógeno desencadenando desde un nivel superior los procesos de maduración, ovulación, desove y espermiación. De esta forma se proporciona una mayor integración de las hormonas necesarias para la reproducción (8). En busca de mejorar el desempeño reproductivo, la optimización de las técnicas reproductivas y disminución de los costos, se han empleado dosis desde los 4.4 y $8 \mathrm{mg} / \mathrm{kg}$ de peso corporal utilizando EHC y desde los 0.050 y $0.75 \mathrm{~mL} / \mathrm{kg}$ con Ovaprim $\AA(9,10)$.

El objetivo de este estudio fue evaluar el efecto de inductores hormonales durante la reproducción de nicuro Pimelodus blochii. 


\section{MATERIALES Y MÉTODOS}

Sitio de estudio. El estudio se realizó en la estación piscícola Piedra Pintada, adscrita a la Central de Cooperativas de Caficultores del Huila CENTRACAFE, localizada a $3.3 \mathrm{Km}$ del municipio de Aipe-Huila (Colombia), a $430 \mathrm{~m}$ sobre el nivel del mar, con pluviosidad de $1146.3 \mathrm{~mm}$ anuales, temperatura ambiente promedio de $28.1^{\circ} \mathrm{C}$, temperatura promedio del agua $27^{\circ} \mathrm{C}$ y humedad relativa del $67 \%$.

Material biológico. Los ejemplares de nicuro fueron tomados del plantel de reproductores mantenidos en estanques en tierra, los cuales fueron sometidos inicialmente a un período de manipulación mensualmente, buscando cierto grado de "acostumbramiento", alimentados con una ración de $25 \%$ de proteína bruta, una vez al día, todos los días. Mensualmente se evaluó su maduración sexual. Para los procesos experimentales fueron preseleccionadas hembras por sus características externas como vientre abultado y papila genital ligeramente dilatada y enrojecida $(11,12)$. Para la selección final de las hembras se evaluó la ubicación de la vesícula germinal o núcleo, estimando posiciones como central, en migración y periférico, así como los deformes (atrésicos). Para el caso de los machos la identificación y selección se realizó por medio de una observación más directa, colocando una alícuota de la muestra $(10 \mu \mathrm{L})$ sobre una lámina portaobjetos montada en un microscopio óptico (10X de magnificación (Olympus, CX21, Japón), se determino la presencia o ausencia de espermatozoides debido a que no se muestra una viscosidad en las muestras colectadas, lo cual puede ser un factor de error en la selección. Los individuos seleccionados fueron trasladados a piletas circulares de cemento con una altura de $30 \mathrm{~cm}$ de columna de agua, donde permanecieron por lo menos $36 \mathrm{~h}$ antes de iniciar el proceso de inducción y hasta el final del experimento. Allí se identificaron por medio de microchips (Allflex, microchips inyectable, Francia) previamente implantados debajo de la aleta dorsal, registrando su peso corporal $(\mathrm{g})$ y la longitud total $(\mathrm{cm})$ de cada individuo.

Inducción hormonal. Se evaluaron tres tratamientos, utilizando Extracto de Hipófisis de Carpa (EHC) y una mezcla comercial de análogo superactivo de hormona liberadora de gonadotropina con un bloqueador de los receptores de D2 de dopamina, Ovaprim \& (OVAP: sGnRHa + domperidona). Los tratamientos consistieron en aplicar vía intramuscular $0.25 \mathrm{~mL} / \mathrm{kg}$ OVAP (T1dosis única), $0.5 \mathrm{~mL} / \mathrm{kg}$ de OVAP (T2-dosis única) y $6.25 \mathrm{mg} / \mathrm{kg}$ de EHC (T3), para este último tratamiento la inyección fue dividida en 20 y $80 \%$, con un intervalo de 12 h entre aplicación.

Se utilizó como tratamiento control, hembras inyectadas con solución salina fisiológica, siguiendo el mismo cronograma de los animales tratados. Para el caso de los machos con un peso promedio de $65.6 \pm 20.2 \mathrm{~g}$, se les aplicó una dosis única $(100 \%)$ de $0.75 \mathrm{~mL} / \mathrm{kg}$ de Ovaprim ${ }^{\circledR}$.

Período de latencia. Para conocer el intervalo de tiempo entre la ultima inyección decisiva y la ovulación, se calculó el período de latencia ( ${ }^{\circ}$ h), tiempo transcurrido desde la aplicación de la última inyección hormonal hasta el momento de la ovulación y las hembras ovuladas se consideraron cuando los ovocitos salían fluidamente mediante presión anterio-posterior de la cavidad celómica. Considerando de esta manera que las hembras respondieron positivamente a la inducción hormonal.

Durante el experimento se midieron parámetros fisicoquímicos del agua, como temperatura $\left({ }^{\circ} \mathrm{C}\right)$, oxígeno disuelto $(\mathrm{mg} / \mathrm{L}), \mathrm{pH}$, conductividad eléctrica $(\mu \mathrm{s} / \mathrm{cm})$ y sólidos disueltos $(\mathrm{mg} / \mathrm{L})$, empleando una sonda multiparamétrica (YSI 556, EUA).

Obtención de los gametos. Previo a la extracción de los gametos, los animales fueron tranquilizados por inmersión en una solución de Metanosulfonato de Tricaina (MS222, Argent Laboratories, EUA) a una concentración de $90 \mathrm{mg} / \mathrm{L}$. Una vez observados los síntomas de anestesia como la pérdida del eje nado y disminución de los movimientos operculares, se retiraron de la solución, se secó cuidadosamente en el área ventral y se realizó presión manual en sentido cráneo caudal. Los ovocitos fueron recolectados en recipientes plásticos, mientras que el semen fue colectado con la ayuda de una micropipeta para aquellos animales que espermiaron.

Evaluación reproductiva. El desempeño reproductivo fue evaluado mediante el índice de ovulación (hembras ovuladas/hembras tratada). Se estimó la fecundidad absoluta ( $\mathrm{Fa}$ ) considerando el número de ovocitos desovados por hembra (ovocitos/hembra). Luego se calculó la fecundidad relativa $(\mathrm{Fr})$ en función del número de ovocitos desovados por gramo de peso.

La fecundación se realizó en seco y seis horas post-fecundación (HPF) se determinó la tasa de fertilidad, en la fase del cierre del blastoporo.

Antes de la aplicación del tratamiento hormonal, para determinar el diámetro de los ovocitos en 
maduración final, se midieron 50 ovocitos de cada hembra, utilizando una reglilla adaptada a un estereoscopio (NIKON, SMZ800, Japón). Igualmente, los ovocitos postinducción se les midió su diámetro.

Análisis de resultados. Los resultados fueron expresados como media \pm error estándar de la media (SEM). Para determinar los efectos de los tratamientos, los resultados fueron sometidos a un análisis de varianza (ANOVA). Inicialmente los datos fueron validados mediante los supuestos de homogeneidad de varianza y normalidad. Se utilizó como prueba de comparación TukeyKramer, con un nivel de significancia de $p<0.05$. Los procedimientos estadísticos fueron realizados empleando el software GraphPad InStat versión 3.06 para Windows.

\section{RESULTADOS}

La temperatura a la cual ocurrieron los desoves fue de $26.5 \pm 0.1^{\circ} \mathrm{C}$, $\mathrm{pH}$ de $7.7 \pm 0.1$, conductividad eléctrica de $315.7 \pm 0.5 \mu \mathrm{s} / \mathrm{cm}$ y promedio de sólidos disueltos de $157.6 \pm 0.2(\mathrm{mg} / \mathrm{L})$. La ovulación ocurrió en promedio para el T1 a las

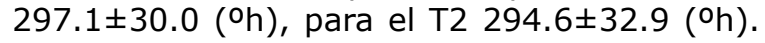
El menor tiempo de respuesta observado fue para el T3 (Tabla 1) con un valor promedio de $247.3 \pm 13.1\left({ }^{\circ} \mathrm{h}\right)$, al comparar esta variable entre los tratamientos no se observó diferencia estadística significativa ( $p>0.05)$.

El estudio demostró que el nicuro respondió positivamente a la inducción hormonal bajo los dos inductores hormonales evaluados. En todos los tratamientos se obtuvieron hembras ovuladas, donde los mayores índices de ovulación fueron obtenidos con Ovaprim ${ }^{\circledR}(\mathrm{T} 1$ y 2$)$ con 36.4 y $50 \%$, respectivamente. El menor índice de ovulación se presentó al utilizar EHC en dosis de $6.25 \mathrm{mg} / \mathrm{kg}$ correspondiente al T3 (20\%). El volumen seminal colectado por los machos inducidos fue muy bajo, menor a $50 \mu \mathrm{L}$, dificultando los procesos de fecundación en seco. En ciertos casos no se observó la presencia de semen por presión abdominal, siendo necesario el sacrificio de estos.
La Fa, estimada en este estudio a partir de los ovocitos obtenidos por único estrujamiento, fue entre 828 y 10743 ovocitos/ hembra con un peso promedio entre $28.9 \pm 4.3$ y $50.4 \pm 6.8 \mathrm{~g}$, que para este caso la mayor Fa fue para el T1 10743.1 ovocitos/hembra (Tabla 1).

Por su parte la $\mathrm{Fr}$, promedio del nicuro osciló entre 1161 y 2070 ovocitos/g, correspondientes a T2 y T3, respectivamente $(p>0.05)$.

El diámetro promedio de los ovocitos preinducción y postinducción fue de $736.3 \pm 13.1$ y $1172.1 \pm 4.1$ $\mu \mathrm{m}$, respectivamente.

Las tasas de fecundación obtenidas en este estudio fueron mayores al $50 \%$, para el caso del tratamiento 1 y 2 , con valores de 74.5 y $32.7 \%$, respectivamente.

\section{DISCUSIón}

Los ovocitos maduros de nicuro muestran un color amarillo, mientras que el semen es incoloro, sin presencia de cortejo. El estudio demostró que el nicuro respondió a la inducción hormonal con Ovaprim ${ }^{\circledR}$ y EHC; sin embargo, en dosis de 6.25 $\mathrm{mg} / \mathrm{kg}$ de EHC (T3), representó el porcentaje más bajo, respondiendo sólo una hembra. Dosis similares han sido aplicadas en otros bagres como el blanquillo (Sorubim cuspicaudus) por Muñoz y Atencio (10) en una sola dosificación para el caso de Ovaprim, obteniendo un índice de ovulación de $66.7 \%$ al utilizar $0.25 \mathrm{~mL} / \mathrm{kg}$ de Ovaprim, siendo mayor al ser comparado en este estudio. Autores como Aya y Arias (13), observaron un periodo de latencia mas corta en tigrito (Pimelodus pictus) tratados con $\mathrm{EHC}$ que en peces tratados con otras hormonas, lo cual concuerda con lo observado en este estudio para el caso del tratamiento 3 y 4. Esto puede ser explicado por el hecho de que la liberación de GnRH de la hipófisis y su respuesta en el ovario es un proceso secuencial, mientras que en los peces inyectados con EHC, la respuesta del ovario a la $\mathrm{LH}$ exógena es un proceso simple $(14,15)$. Por otro lado la presencia de $\mathrm{GnRH}$ en presentación comercial

Tabla 1. Resultados obtenidos con los diferentes protocolos de inducción de la ovulación y el desove de hembras de nicuro (Pimelodus blochii) con Ovaprim $®$ y EHC. Datos mostrados como media \pm error estándar de la media (SEM) $(p>0.05)$.

\begin{tabular}{|c|c|c|c|c|c|c|c|}
\hline Tto & Peso (g) & Longitud (cm) & $n^{1}$ & Periodo de latencia $\left({ }^{\circ} \mathrm{h}\right)$ & $\mathbf{F a}$ & $\mathbf{F r}$ & TF (\%) \\
\hline 1 & $50.4 \pm 6.8$ & $34.5 \pm 16.3$ & $11 / 4$ & $297.1 \pm 30.0$ & $10743.1 \pm 9641.1$ & $1903 \pm 556.8$ & $74.6 \pm 0.0$ \\
\hline 2 & $55.8 \pm 9.1$ & $32.6 \pm 13.9$ & $12 / 6$ & $294.6 \pm 32.9$ & $2086.5 \pm 873.8$ & $1161.2 \pm 337$ & $32.7 \pm 16.7$ \\
\hline 3 & $28.9 \pm 4.3$ & $16.2 \pm 0.9$ & $10 / 2$ & $247.3 \pm 13.1$ & $828 \pm 288$ & $2070 \pm 720$ & 0 \\
\hline Cnt & $35.8 \pm 8.0$ & $16.9 \pm 1.5$ & $9 / 0$ & ----- & ---- & ---- & ---- \\
\hline
\end{tabular}

Tto = tratamiento, Cnt = tratamiento control; ${ }^{1}$ Hembras tratadas/hembras ovuladas; Fa $=$ fecundidad absoluta; Fr= fecundidad relativa; $\mathrm{TF}=$ tasa de fertilidad 
como el Ovaprim $®$, proporciona un estímulo más equilibrado de eventos reproductivos $y$, presumiblemente una mejor integración de estos eventos con otras funciones fisiológicas, por directa o indirectamente que afecta a la liberación de otras hormonas necesarias para el éxito de la reproducción inducida hormonalmente (8).

El porcentaje de respuesta ovulatoria, tiempo de latencia y porcentaje de fertilización en barbilla (Rhamdia sebae cf), utilizando $5.5 \mathrm{mg} / \mathrm{kg}$ de EHC en dosis de 10 y $90 \%$, en intervalo de $12 \mathrm{~h}$, fue de $9 / 12,7.00 \pm 0.04$ h y $60 \pm 24 \%$ (16). El período de latencia de la ovulación fue de $257.6 \pm 8.50 \mathrm{~h}$ para el caso del bagre rayado (Pseudoplatystoma fasciatum), a una temperatura promedio de $25.8 \pm 0.9^{\circ} \mathrm{C}(16)$, con una fertilidad observada a las $6 \mathrm{~h}$ postseminación de $81.1 \pm 14.3 \%$. Esto valores para el caso del porcentaje de fertilidad es mayor a lo observado en este estudio, dentro de las causas a la posible bajas en la tasa de fertilización se puede atribuir a la condición nutricional de los reproductores, a pesar de una alimentación constante en el cautiverio, donde cabe anotar que se trataban de individuos que provenían del medio natural bajo siete meses de adaptación a las condiciones del cautiverio, sumado al bajo o no presencia de volumen espermático en algunos casos.

La mayor dificultad que presenta el proceso de reproducción inducida de Pimelodus blochii, encontrado en este estudio reside en la determinación de la madurez de los machos por la no presencia de liberación espontanea de semen aún después del tratamiento hormonal. A pesar, de que en las diferentes especies de peces no necesitan de tratamiento hormonal para inducir la espermiación, no obstante, este proceso optimiza el manejo del semen, lo cual facilita los procesos de fertilización artificial. Al igual que el nicuro, en especies como Clarias gariepinus $(17,18)$, Ictalurus punctatus (19) y Leiarius marmoratus (20) no hay liberación espontanea de semen aún después de un tratamiento de inducción hormonal, siendo necesario el sacrificio de los machos para obtener el semen, con la necesidad e importancia de poder lograr la disminución de esta práctica y conseguir programas de reproducción económicamente viables $(19,21)$.

Al comparar los costos con estas dos sustancias, es importante considerar las diferencias de precios existentes en el mercado, donde el EHC presenta un incremento mayor al $80 \%$ con respecto al Ovaprim $\AA$, de acuerdo a sus presentaciones existentes ( $1 \mathrm{~g}$ y $10 \mathrm{~mL}$, respectivamente). De acuerdo al presente estudio la dosificación de $0.25 \mathrm{~mL} / \mathrm{kg}$ se observa un ahorro del alrededor del $42 \%$ con respecto a la dosis empleada de $6.25 \mathrm{mg} / \mathrm{kg}$ de EHC, resultando más económico en la inducción de nicuro $P$. blochii.

En conclusión el uso de inductores hormonales puede ser efectivo, sugiriendo que el Ovaprim $\mathbb{R}$ en dosis única de 0.25 y $0.5 \mathrm{~mL} / \mathrm{kg}$ es suficiente para garantizar la reproducción inducida de Pimelodus blochii, además de ser una opción mas económica que el EHC.

\section{Agradecimientos}

Al Servicio Nacional de Aprendizaje SENA, a la Federación Colombiana de Acuicultores FEDEACUA, por la financiación de la investigación y a la Central de Cooperativas de Caficultores del Huila CENTRACAFE, por su apoyo en el desarrollo del presente estudio.

\section{REFERENCIAS}

1. Maldonado OJA, Ortega A, Usma JS, Galvis G, Villa FA, Vásquez I et al. Peces de los Andes de Colombia. Bogotá D. C., Colombia: Instituto de Investigación de Recursos Biológicos Alexander von Humboldt; 2005.

2. Instituto Nacional de Pesca y AcuiculturaINPA. Boletín estadístico y pesquero Colombiano. Bogotá, Colombia: Ministerio de Agricultura; 2002.
3. Usma JS, Valderrama M, Escobar M, Ajiaco RE, Villa F, Castro F et al. Peces dulceacuícolas migratorios en Colombia. En: Plan Nacional de las Especies Migratorias. WWF. Bogotá D.C.: Dirección de ecosistemas; 2009.

4. Prada-Pedreros S. Biología, dinâmica populacional e avaliaçao do estoque de mandiamarelo Pimelodus meculatus Lacépede, 1803, (Ostariophysi: Siluriformes, Pimelodidae) da bacia do alto e medio Rio Paraná, Brasil. [Tese doctoral]. Rio Claro, Brasil: Universidade Estadual Paulista, Instituto de Biociências; 2003. 
5. Rodríguez JA. Maduración gonadal del nicuro (Pimelodus blochii) en cautiverio. En: IV Seminario Internacional de Acuicultura, Bogotá: Universidad Nacional de Colombia; 2004.

6. López-Casas S, Jiménez-Segura LF. Reproducción y hábitos alimenticios del nicuro Pimelodus blochii (Valenciennes, 1840) (Pisces: Pimelodidae), en la ciénaga de Cachimber, río Magdalena, Colombia. Actual Biol 2007; 29:1-13.

7. Leonardo AFG, Romagosa E, Batlouni SR, Borella MI. Occurrence and significance of ovarian and follicular regression in cachara Pseudoplatystoma fasciatum (Linnaeus, 1766): a histology approach. Arq Bras Med Vet Zootec 2006; 58: 831-840.

8. Zohar Y, Mylonas Constantinos C. Endocrine manipulations of spawning in cultured fish: from hormones to genes. Aquacult 2001; 197: 99-136.

9. Atencio GV. Producción de alevinos de especies nativas. Rev MVZ Córdoba 2001; 6(1): 9-14.

10. Muñoz BRJ, Atencio GV. Evaluación de la reproducción inducida del blanquillo (Sorubim cuspicaudus Littmann, Burr \& Nass, 2000) con Ovaprim ${ }^{\circledR}$. Rev. MVZ Córdoba 2003; 8(2):333-334.

11. Pardo-Carrasco S. Reproducção induzida do yamú Brycon siebenthalae (Pisces: Characidae). [Tese maestría]. Universidade Federal de Santa Catarina; 2001.

12. Zaniboni-Filho E, Nuñer AP. Fisiologia da reprodução e propagação artifi cial dos peixes. En: Cyrino JEP, Urbinati EC, Fracalossi DM, Castagnolli N. (Eds.). Tópicos especiais em piscicultura de água doce tropical in-tensiva. Brasil: Sociedade Brasileira de Aqüicultura e Biologia Aquática; 2004.

13. Aya $\mathrm{BE}$, Arias $\mathrm{CJ}$. Reproducción inducida de Pimelodus pictus con extracto de hipófisis de carpa (EHC) y Ovaprim $\AA$. Rev MVZ Córdoba $2011 ; 16(1): 2317-2323$.

14. Kucharczyk D, Kujawa R, Mamcarz a, Wyszomirska E, Ulikowsky D. Artificial spawning of ide (Leuciscus idus) under controlled conditions. EJPAU [en línea] 1999 [fecha de acceso noviembre de 2007]; URL disponible en: http://www.ejpau.media.pl/ volume2/issue2/fisheries/art-05.html
15. Díaz SE, Arias CJA, Aya BE. Comparación del Ovaprim y del extracto de hipófisis de carpa (EHC) en la inducción y ovulación y desove de Rhamdia sebae of (Pisces Pimelodidae). En: II Congreso Colombiano de Acuicultura. $X$ Jornada de Acuicultura IALL, Villavicencio: Universidad de los Llanos; 2004.

16. Mira T, Castro SR, Medina-Robles VM, Murillo $\mathrm{RP}$, Otero-Paternina AM, Ramírez-Merlano JA et al. Ensayos preliminares de reproducción inducida de bagre rayado Pseudoplatystoma fasciatum con extracto de hipófisis de carpa. En XIII Jornada de Acuicultura. Villavicencio: Universidad de los Llanos; 2007.

17. Viveiros ATM, Fessehave $Y$, Ter Veld M, Schulz RW, Komen J. Hand-stripping of semen and semen quality after maturational hormone treatments, in African catfish Clarias gariepinus. Aquacult 2002; 213:373-386.

18. Brzuska E. Artificial propagation of African catfish (Clarias gariepinus): differences between reproduction effects after stimulation of ovulation with carp pituitary homogenate or GnRH-a and dopaminergic inhibitor. Czech J Anim Sci 2003; 48(5):181-190.

19. Christensen JM, Tiersch TR. Cryopreservation of chanel catfish sperm: effects of cryopreservation exposure time, cooling rate, thawing condictions, and maleto-male variation. Theriogenology 2005; 63:2103-2112.

20. Castillo Jiménez AM, Ramírez Lesmes R, Rodríguez Pulido JA. Ensayos de reproducción y alevinaje en Yaque Leiarius marmoratus (Gill, 1870) (Pisces: Siluriformes: Pimelodidae) en la Orinoquia Colombiana. En: VI Seminario Internacional de Investigaciones Acuícolas, Bogotá: Universidad Nacional de Colombia, 2003.

21. Chowdhury I, Joy KP. Seminal vesicle and testis secretions in Heteropneustes fossilis (Bloch): composition and effects on sperm motility and fertilization. Aquacult 2001; 193:355-371. 